\title{
Neuroimaging findings of congenital Zika virus infection: a pictorial essay
}

\author{
Mohammad Zare Mehrjardi ${ }^{1}$ (D) Andrea Poretti $^{2} \cdot$ Thierry A. G. M. Huisman $^{2} \cdot$ \\ Heron Werner ${ }^{3} \cdot$ Elham Keshavarz $^{4} \cdot$ Edward Araujo Júnior $^{5}$
}

Received: 25 November 2016 / Accepted: 27 December 2016 / Published online: 10 January 2017

(C) Japan Radiological Society 2017

\begin{abstract}
Zika virus (ZIKV) is a mosquito-borne arbovirus from the Flaviviridae family. It had caused several epidemics since its discovery in 1947 , but there was no significant attention to this virus until the recent outbreak in Brazil in 2015. The main concern is the causal relationship between prenatal ZIKV infection and congenital microcephaly, which has been confirmed recently. Moreover, ZIKV may cause other central nervous system abnormalities such as brain parenchymal atrophy with secondary ventriculomegaly, intracranial calcification, malformations of cortical development (such as polymicrogyria, and lissencephalypachygyria), agenesis/hypoplasia of the corpus callosum, cerebellar and brainstem hypoplasia, sensorineural hearingloss, and ocular abnormalities as well as arthrogryposis in the infected fetuses. Postnatal (acquired) ZIKV infection usually has an asymptomatic or mildly symptomatic course, while prenatal (congenital) ZIKV infection has a more severe course and may cause severe brain anomalies that are described as congenital Zika syndrome. In this
\end{abstract}

Mohammad Zare Mehrjardi

zare@sbmu.ac.ir

Andrea Poretti

aporett1@jhmi.edu

Thierry A. G. M. Huisman

thuisma1@jhmi.edu

Heron Werner

heronwerner@hotmail.com

Elham Keshavarz

elhamkeshavarz@yahoo.com

Edward Araujo Júnior

araujojred@terra.com.br

1 Department of Radiology, Shohada Tajrish Hospital, Shahid

Beheshti University of Medical Sciences, Tehran, Iran pictorial essay, we aim to illustrate the prenatal and postnatal neuroimaging findings that may be seen in fetuses and neonates with congenital Zika syndrome, and will discuss possible radiological differential diagnoses. A detailed knowledge of these findings is paramount for an early correct diagnosis, prognosis determination, and counseling of the affected children and families.

Keywords Zika virus (ZIKV) · Congenital · Radiology · Neuroimaging $\cdot$ Microcephaly

\section{Introduction}

Zika virus (ZIKV) is a mosquito-borne arbovirus, which was first discovered in the mid-twentieth century [1]. Although some previous epidemics have been reported, the ZIKV gained attention after the recent outbreak in Brazil in 2015. The main concern is the causal relationship between congenital ZIKV

2 Section of Pediatric Neuroradiology, Division of Pediatric Radiology, Russell H. Morgan Department of Radiology and Radiological Science, The Johns Hopkins University School of Medicine, Baltimore, MD, USA

3 Department of Radiology, Clínica de Diagnóstico por Imagem (CDPI), Rio de Janeiro, RJ, Brazil

4 Department of Radiology, Mahdieh Women's Hospital, Shahid Beheshti University of Medical Sciences, Tehran, Iran

5 Department of Obstetrics, Paulista School of Medicine, Federal University of São Paulo (EPM-UNIFESP), São Paulo, SP, Brazil 
infection and adverse pregnancy and birth outcome, particularly congenital microcephaly and other severe brain anomalies [2]. This association suggests that ZIKV infection is less benign than initially thought, and led the World Health Organization (WHO) to declare Zika a "Public Health Emergency of International Concern" in February 2016 [3].

ZIKV has been shown to disrupt the normal brain development most likely by direct cellular injury as ZIKV RNA and live virus have been identified in the brain tissue of fetuses and neonates with microcephaly due to congenital Zika infection [4]. ZIKV can theoretically disrupt the normal fetal brain development at any stage, explaining the spectrum of neuroimaging findings reported so far in congenital Zika syndrome. Animal models, however, have shown that neural progenitor cells are the primary target of ZIKV in the developing brain, explaining the severe brain anomalies seen on neuroimaging studies. Immature neurons may be also affected, but to a milder degree.

Disruptions of brain development are defined as a congenital morphologic brain anomaly due to the breakdown in a brain structure that had a normal developmental potential [5]. Disruptions may be caused by insults such as prenatal infection, hemorrhage, or ischemia and are non-inherited lesions with very low recurrence risk in the next pregnancies. It is important to differentiate disruptions from malformations, which are congenital morphologic brain anomalies due to an alteration in the primary brain developmental program caused by a genetic defect. Despite disruptions of brain development, malformations may be associated with an increased recurrence risk due to genetic basis.

In this pictorial essay, we aim to illustrate the currently recognized spectrum of neuroimaging findings in congenital Zika infection that may be seen using different imaging modalities such as ultrasound, computed tomography (CT), and magnetic resonance imaging (MRI). Familiarity with these findings is paramount for an early accurate diagnosis, prognosis determination, and counseling of the affected children and families.

\section{Prenatal neuroimaging}

\section{Ultrasound (Fig. 1)}

Prenatal ultrasound examination is an inexpensive, easyto-access, and safe method for screening pregnant women residing in or traveling to endemic areas. The most common finding in congenital Zika syndrome is microcephaly (i.e., head circumference (HC) more than 2 standard deviations below the mean that is appropriate for the gestational age). Fetal HC can be easily measured by ultrasound. However, it should be noted that there are also reports of severely affected fetuses with normal $\mathrm{HC}$ at birth.

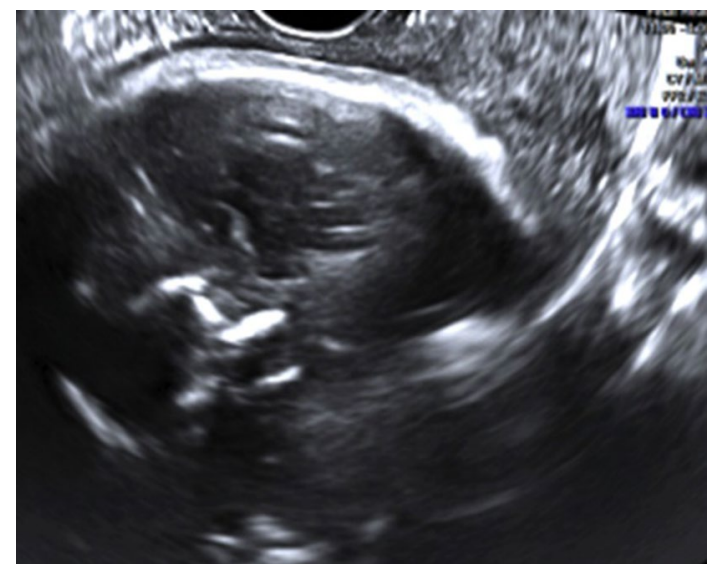

Fig. 1 Fetal brain ultrasound in congenital ZIKV infection. Transvaginal ultrasound shows brain parenchymal calcifications in a 37-week fetus. Note that brain assessment is limited by the ossified skull shadowing artifact

Other findings of congenital ZIKV infection that may be seen in prenatal ultrasound examination include parenchymal atrophy with secondary ventriculomegaly, subependymal pseudocysts, parenchymal calcification, agenesis/hypoplasia of the corpus callosum, cerebellar and brainstem hypoplasia, and ocular abnormalities [6-11].

\section{Magnetic resonance imaging (Fig. 2)}

Fetal brain magnetic resonance imaging (MRI) is helpful for better delineation of brain abnormalities, and may be performed when fetal ultrasound shows brain abnormalities suggestive of ZIKV infection.

Findings are similar to prenatal ultrasound. However, malformations of cortical development such as polymicrogyria, opercular dysplasia, and lissencephaly-pachygyria as well as white-matter and cortical changes such as abnormal myelination, and cortical laminar necrosis are better evaluated by MRI. Compared to fetal ultrasound, prenatal MRI is, however, less sensitive in detecting discrete/dispersed intraparenchymal calcifications $[7,8,12]$.

\section{Postnatal neuroimaging}

\section{Ultrasound (Fig. 3)}

Ultrasound may be used as an inexpensive and safe modality for the first-line investigation of suspected neonates. Both brain parenchyma and the ventricular system can be evaluated by ultrasound.

Ultrasound may show parenchymal calcifications that are typically located at or just below the corticomedullary junction, parenchymal atrophy with secondary 
Fig. 2 Fetal brain MRI in congenital ZIKV infection. A smooth and thin cortical ribbon, thin corpus callosum, and enlarged ventricular system are seen on axial T2-weighted (a), and sagittal T2-weighted (b) MR images of the same patient as Fig. 1
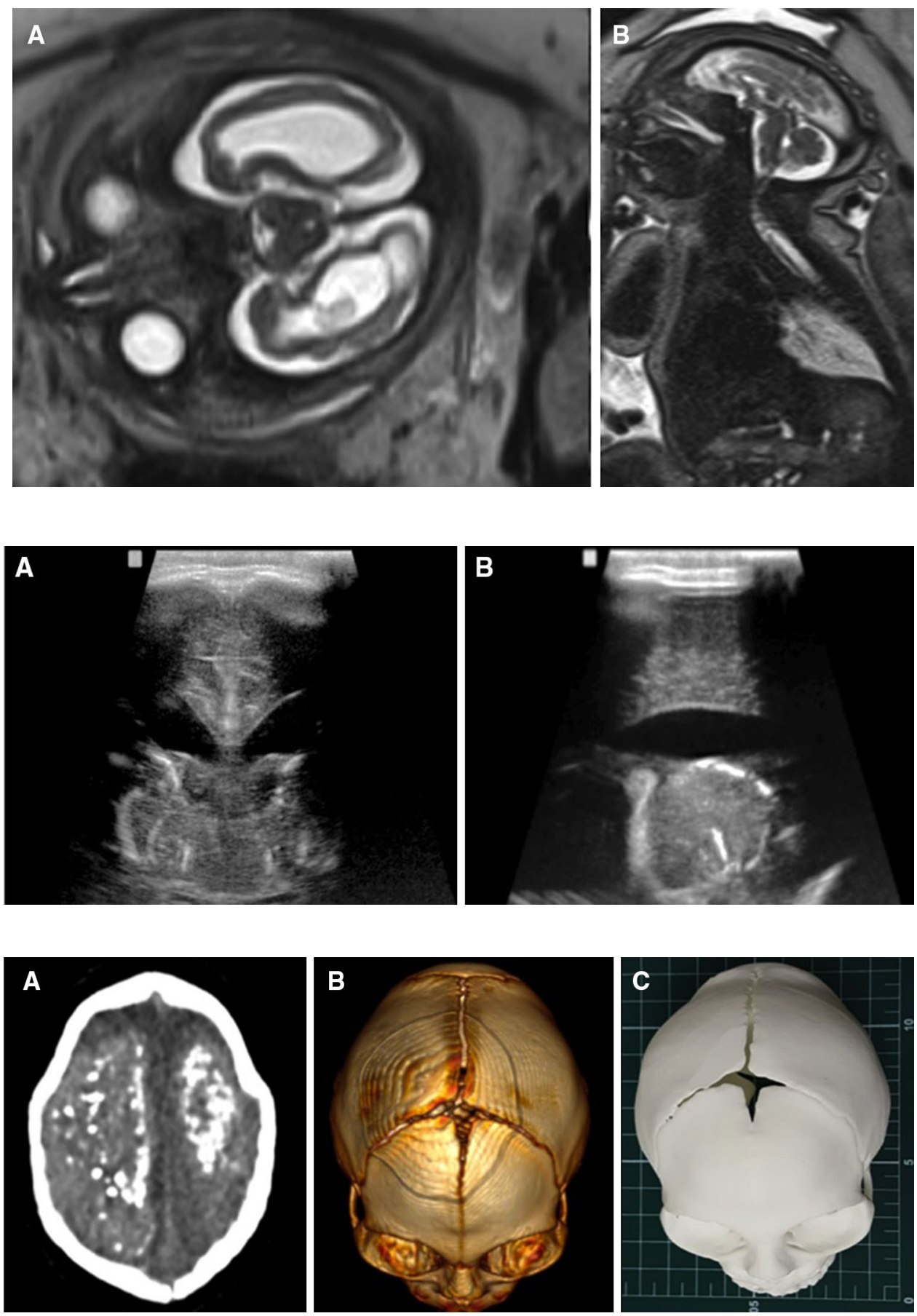

Fig. 4 Head CT scan in congenital ZIKV infection. Diffuse punctate calcifications are noted mostly at the corticomedullary junction with a band-like distribution (a). Microcephaly and a small anterior fontanel are seen on the skull 3D reconstruction (b), and physical modeling (c) of the same patient ventriculomegaly, absence/thinning of the corpus callosum, subependymal cysts, severe malformations of cortical development, and cerebellar and brainstem hypoplasia [7, 8].

\section{Computed tomography (Fig. 4)}

Computed tomography (CT) scan is a sensitive modality for evaluating parenchymal calcifications. It also shows associated skull deformities well, particularly while using 3-dimensional (3D) reconstructions.

Parenchymal calcifications are the most common intracranial finding in congenital ZIKV infection and have been reported in almost all affected patients so far. Calcifications are most likely dystrophic in nature, and are most commonly punctate in shape with a band-like distribution, and located at or just below the corticomedullary junction within the frontal and parietal lobes. Basal ganglia 

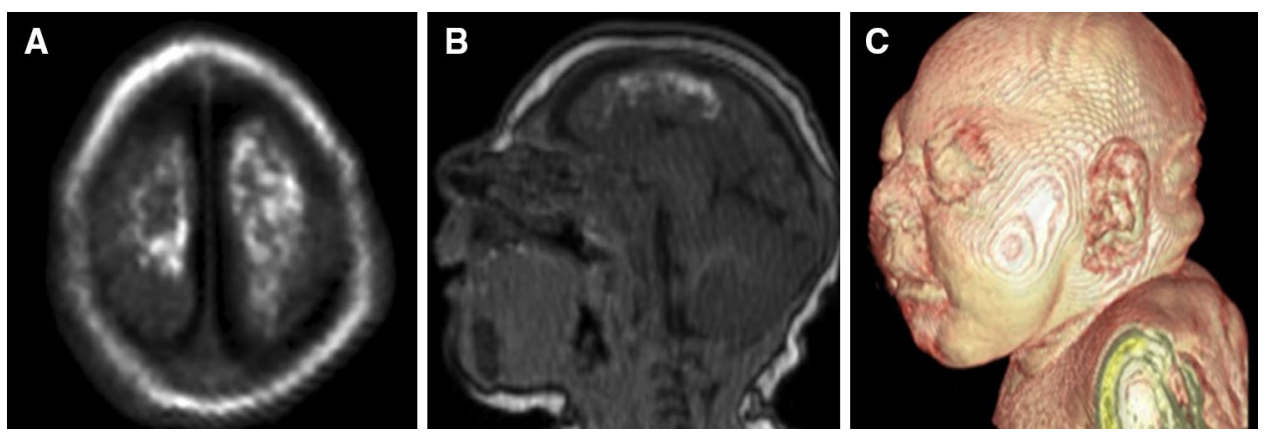

Fig. 5 Brain MRI in congenital ZIKV infection. Brain MRI shows diffuse parenchymal calcification as hyperintense foci on an axial T1-weighted image, which indicates that the calcified foci are newly formed and contain a low amount of calcium (less than $30 \%$ concen-

(in about $65 \%$ of cases), thalami (in about $40 \%$ of cases), and cerebellum are less common locations that may become calcified. The exact location of calcifications is not always easy to determine because of the marked cortical thinning.

Severe craniofacial disproportion with depression of the frontal and parietal bones, cranial bone collapse, overlapping sutures, a pointed appearance of the occipital and frontal regions, and small fontanels may be best seen on bone window and 3D reconstructions of head CT scans. These findings are most likely secondary to loss of brain volume and decrease in intracranial pressure.

Microcephaly, parenchymal atrophy, ventriculomegaly, malformations of cortical development (seen as a "smooth" cortical ribbon), agenesis/hypoplasia of the corpus callosum, and white-matter hypodensity due to abnormal myelination are other findings that may be observed on head CT scans $[7,8,13-15]$.

\section{Magnetic resonance imaging (Fig. 5)}

Magnetic resonance imaging (MRI) is the neuroimaging technique of first choice to evaluate the pediatric brain because of the high spatial and contrast resolution that results in a high sensitivity and specificity for detecting brain abnormalities such as malformations of cortical development, and white-matter abnormalities.

In congenital Zika infection with microcephaly, malformations of cortical development are present in $95-100 \%$ of the patients, affect the whole brain in the majority of the cases, and are mostly characterized by marked cortical thinning and an abnormal gyral pattern most consistent with polymicrogyria. Pachygyria and heterotopias are less common. Abnormal white-matter signal is present in $88-100 \%$ of the patients and is most likely due to delayed myelination or dysmyelination. tration) (a). Multiple calcifications and smooth cortical ribbon are seen on a sagittal T1-weighted image (b). Microcephaly and craniofacial disproportion are seen on MRI 3D reconstruction (c)

Ventriculomegaly is present in $85-100 \%$ of the patients, is mostly moderate to severe, and typically involves the whole ventricular system. Septations in the lateral ventricles are reported in 10-30\% of the affected infants, and subependymal cysts may be seen, suggesting the infectious etiology. Callosal abnormalities are seen in 75-94\% of the patients, are mostly characterized by a thin, dysgenetic, hypoplastic or absent corpus callosum, and may be associated with an abnormal rotation of the hippocampi and thickened fornices. Cerebellar hypoplasia is present in $27-82 \%$ of the infants and usually globally involves the cerebellum. The cerebellar hemispheres have mostly a symmetric involvement, although unilateral cerebellar hypoplasia has been reported. A brainstem hypoplasia is present in $21-70 \%$ of the children and involves mostly the pons. MRI may also detect spinal cord involvement as thinning of the spinal cord and reduction in size of the ventral roots. The application of susceptibility weighted imaging increases the sensitivity of MRI in detecting intracranial calcifications. Large choroid plexus is another finding that may be observed on brain MRI [7, 8, 13].

\section{Neuroimaging findings in children with congenital ZIKV infection without microcephaly}

Brain anomalies have been recently reported in 13 children with congenital ZIKV infection, but without congenital microcephaly [16]. Neuroimaging findings in these children included malformations of cortical development (more predominantly affecting the anterior brain), calcifications at the corticomedullary junction, decreased brain volume, and ventriculomegaly in all children. Hypoplasia of the corpus callosum, cerebellum, and brainstem was seen in some of the patients. This report suggests that there may be a wide spectrum of findings associated with congenital ZIKV 
Fig. 6 Radiological differential diagnosis of congenital Zika infection. Mild brain parenchymal atrophy, ventricular dilatation, and periventricular and basal ganglia calcification are seen in two different neonates with congenital cytomegalovirus (CMV) infection in axial CT scans $(\mathbf{a}, \mathbf{b})$. Basal ganglia and white-matter calcifications are noted in a child with AicardiGoutières syndrome on axial brain CT scan (c)
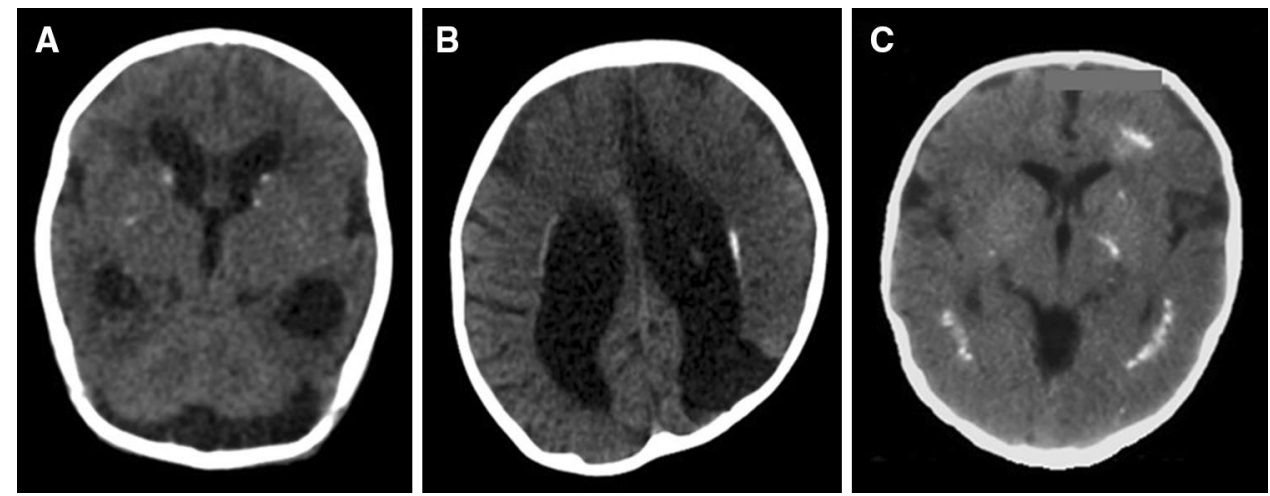

infection and that we know so far the most severe end of this spectrum. Future studies in less symptomatic children with congenital ZIKV infection may reveal the whole spectrum of brain anomalies and neuroimaging findings associated with congenital Zika infection. It is still unknown whether the timing of infection may also play a determining role in the severity of brain anomalies as known for other congenital infections such as cytomegalovirus (early infection causes more severe brain anomalies).

\section{Neuroimaging-based differential diagnosis (Fig. 6)}

Some other congenital infections and genetic disorders may mimic the neuroimaging findings of congenital ZIKV infection.

Prenatal infections such as TORCH infections may disrupt fetal brain development, and result in various brain injuries and congenital brain abnormalities. Congenital cytomegalovirus (CMV) infection has the most similar features to congenital Zika syndrome among TORCH infections. However, the location of calcifications seems to be different in congenital CMV and ZIKV infections: mostly periventricular in CMV infection and mostly at the corticomedullary junction in ZIKV infection. In addition, hepatic, renal, and hematologic abnormalities are uncommon in congenital ZIKV infection (unlike congenital CMV infection).

Various genetic and metabolic disorders may cause microcephaly and/or intracranial calcifications and, hence, mimic congenital ZIKV infection. Aicardi-Goutières syndrome is an autosomal-recessive, immune-mediated disorder caused by mutations in several genes such as TREX1. The location of calcifications (at the corticomedullary junction in congenital ZIKV infection versus in the basal ganglia, periventricular white-matter, and dentate nuclei in Aicardi-Goutières syndrome), and the lack of abnormal cortical development in Aicardi-Goutières syndrome may help to differentiate between these diseases.
Generally, clinical history, specific neuroimaging features, and laboratory studies may help to differentiate congenital ZIKV infection and its neuroimaging mimickers $[4,6,7]$.

\section{Conclusion}

Congenital ZIKV infection may cause several brain abnormalities that may be detected by prenatal and postnatal neuroimaging techniques including ultrasound, CT scan, and MRI. Although these findings are not pathognomonic, the details neuroimaging provide in combination with clinical history, and laboratory studies are important for an early diagnosis, prognosis determination, and counseling of the affected children and families.

Compliance with ethical standards

Conflict of interest There is no conflict of interest.

\section{References}

1. Dick GW, Kitchen SF, Haddow AJ. Zika virus. I. Isolations and serological specificity. Trans R Soc Trop Med Hyg. 1952;46:509-20.

2. Rasmussen SA, Jamieson DJ, Honein MA, Petersen LR. Zika virus and birth defects-reviewing the evidence for causality. N Engl J Med. 2016;374(20):1981-7.

3. Zare Mehrjardi M, Poretti A, Keshavarz E. Neuroimaging findings of Zika virus infection: emphasis of congenital versus acquired aspects. Jpn J Radiol. 2016. doi:10.1007/s11604-0160599-2 (Epub ahead of print).

4. Moore CA, Staples JE, Dobyns WB, et al. Characterizing the pattern of anomalies in congenital Zika syndrome for pediatric clinicians. JAMA Pediatr. 2016. doi:10.1001/jamapediatrics.2016.3982 (Epub ahead of print).

5. Hennekam RC, Biesecker LG, Allanson JE, et al. Elements of morphology consortium. Elements of morphology: general terms for congenital anomalies. Am J Med Genet A. 2013;161A(11):2726-33. 
6. Poretti A, Huisman TA. Neuroimaging findings in congenital Zika syndrome. AJNR Am J Neuroradiol. 2016. doi:10.3174/ ajnr.A4924 (Epub ahead of print).

7. Mehrjardi ZM, Keshavarz E, Poretti A, Hazin AN. Neuroimaging findings of Zika virus infection: a review article. Jpn J Radiol. 2016;34(12):765-70.

8. Werner H, Sodré D, Hygino C, et al. First-trimester intrauterine Zika virus infection and brain pathology: prenatal and postnatal neuroimaging findings. Prenat Diagn. 2016;36(8):785-9.

9. Oliveira Melo AS, Malinger G, Ximenes R, et al. Zika virus intrauterine infection causes fetal brain abnormality and microcephaly: tip of the iceberg? Ultrasound Obstet Gynecol. 2016;47(1):6-7.

10. Carvalho FH, Cordeiro KM, Peixoto AB, et al. Associated ultrasonographic findings in fetuses with microcephaly because of suspected Zika virus (ZIKV) infection during pregnancy. Prenat Diagn. 2016;36(9):882-7.

11. Brasil P, Pereira JP Jr, Moreira ME, et al. Zika virus infection in pregnant women in Rio de Janeiro. N Engl J Med. 2016;375(24):2321-34.
12. Guillemette-Artur P, Besnard M, Eyrolle-Guignot D, et al. Prenatal brain MRI of fetuses with Zika virus infection. Pediatr Radiol. 2016;46:1032.

13. de Fatima Vasco Aragao M, van der Linden V, Brainer-Lima AM, et al. Clinical features and neuroimaging (CT and MRI) findings in presumed Zika virus related congenital infection and microcephaly: retrospective case series study. BMJ. 2016;353:11901.

14. Hazin AN, Poretti A, Turchi Martelli CM, et al. Computed tomographic findings in microcephaly associated with Zika virus. $\mathrm{N}$ Engl J Med. 2016;374(22):2193-5.

15. Werner H, Fazecas T, Guedes B, et al. Intrauterine Zika virus infection and microcephaly: correlation of perinatal imaging and three-dimensional virtual physical models. Ultrasound Obstet Gynecol. 2016;47(5):657-60.

16. van der Linden V, Pessoa A, Dobyns W, et al. Description of 13 infants born during October 2015-January 2016 with congenital Zika virus infection without microcephaly at birth - Brazil. MMWR Morb Mortal Wkly Rep. 2016;65(47):1343-48. 